\title{
Intraoperative gastroscopy to determine proximal resection margin during totally laparoscopic gastrectomy for patients with upper third gastric cancer
}

\author{
Peng Yuan ${ }^{1 \#}$, Yan Yan ${ }^{1 \#}$, Yongning Jia ${ }^{2}$, Jing Wang ${ }^{1}$, Ziyu $\mathrm{Li}^{2}$, Qi Wu ${ }^{1}$ \\ ${ }^{1}$ Key Laboratory of Carcinogenesis and Translational Research (Ministry of Education), Department of Endoscopy, Peking University Cancer \\ Hospital \& Institute, Beijing, China; ${ }^{2}$ Key Laboratory of Carcinogenesis and Translational Research (Ministry of Education), Department of \\ Gastrointestinal Surgery, Peking University Cancer Hospital \& Institute, Beijing, China \\ Contributions: (I) Conception and design: P Yuan, Q Wu; (II) Administrative support: Z Li; (III) Provision of study materials or patients: Y Jia; (IV) \\ Collection and assembly of data: Y Yan; (V) Data analysis and interpretation: J Wang; (VI) Manuscript writing: All authors; (VII) Final approval of \\ manuscript: All authors. \\ "These authors contributed equally to this work. \\ Correspondence to: Ziyu Li, MD; Qi Wu, MD. Peking University Cancer Hospital \& Institute, \#52, Fucheng Road, Haidian District, Beijing, China. \\ Email: wuqi1973@163.com; ligregory369@hotmail.com.
}

Background: In totally laparoscopic gastrectomy (TLG), it is usually difficult to determine the proximal margin. Therefore, the present study evaluated the usefulness of intraoperative gastroscopy for direct marking of the tumor proximal margin during TLG for cancer in the upper third of the stomach.

Methods: This retrospective cohort study included 52 patients with gastric cancer who underwent TLG from January 2018 to May 2020. The proximal margin of tumors was determined by intraoperative gastroscopic methods.

Results: Patients were divided into short $(1 \mathrm{~cm})$ and long $(2 \mathrm{~cm})$ groups according to the distance to the proximal margin of the tumor. Participants consisted of 41 males and 11 females with a median age of 63.5 years. Tumors involving the esophagogastric junction (EGJ) occurred in 27 patients. Siewert type II and III tumors were present in 42 and 10 patients, respectively. The median operative time was 244 min. The long group had a statistically significant lower frequency of positive margin than the short group ( $0 \% v s .17 .4 \%$, $\mathrm{P}=0.033$ ). Total gastrectomy was performed in 35 patients, and 17 patients received proximal gastrectomy. No complications associated with the procedure occurred in any patient.

Conclusions: Intraoperative endoscopic views for tumor proximal localization can be used effectively during TLG for patients with upper third gastric cancer. Our results indicate that a distance of $\geq 2 \mathrm{~cm}$ from the proximal resection margin to the tumor was necessary to achieve a negative resection margin. In the future, this may be used as an alternative to frozen section diagnosis.

Keywords: Intraoperative gastroscopy; totally laparoscopic gastrectomy (TLG); proximal resection margin; gastric cancer

Submitted Jul 15, 2020. Accepted for publication Dec 07, 2020.

doi: 10.21037/jgo-20-277

View this article at: http://dx.doi.org/10.21037/jgo-20-277

\section{Introduction}

In gastric cancer surgery, determination of the resection margin is important for a good prognosis (1). Absence of the tumor at the resection margin is an independent prognostic factor for patients with gastric cancer $(2,3)$. Tumor location can be determined easily in laparotomyor laparoscopy-assisted surgery by palpation or direct view through temporary gastrostomy. Totally laparoscopic 
gastrectomy (TLG) for gastric cancer requires that all procedures are completed using only laparoscopic devices but does not require additional minilaparotomy for specimen resection and anastomosis. Since this type of surgery results in less postoperative pain, rapid postoperative recovery, less blood loss, better cosmetic outcomes, and fewer complications relative to open or laparoscopy-assisted surgery that require laparotomy, more surgeons prefer it (4-6). However, an obstacle to TLG for gastric cancer is tumor localization without direct visualization or palpation to determine the area of stomach resection necessary, especially for patients with adenocarcinoma of the esophagogastric junction (EGJ). In such situations, some investigators use endoscopic tattooing or clipping before surgery (7-16). Unfortunately, both methods of marking present some difficulty. In endoscopic tattooing, the amount of indocyanine green or carbon nanoparticles applied and the depth of injection are critical to creating an ideal surgical site. Excessive staining obscures the precise location of the tumor, whereas accidental intraperitoneal injection may cause excessive spread of the dye, creating an unclear surgical area. Preoperative endoscopic clipping, on the other hand, requires the surgeon to palpate clips to identify the tumor location during surgery. In laparoscopic surgery, the ability to palpate the stomach is lost, and it is sometimes difficult to palpate clips located at the EGJ, even through a minilaparotomy wound. Furthermore, preoperative gastroscopy can be inconvenient for patients.

TLG has become more commonly used for intracorporeal anastomosis performed under pneumoperitoneum $(17,18)$. In the process of TLG, it is usually difficult to determine the proximal margin of the tumor, even for advanced gastric cancer, and frozen section analysis is necessary for intraoperative margin assessment. At present, many reports have focused on how to mark margins in distal gastric cancer by endoscopy during distal gastrectomy. The present cohort study evaluated the usefulness of intraoperative gastroscopy for direct marking of the proximal margin of tumors during TLG for gastric cancer in the upper third of the stomach.

We present the study in accordance with the STROBE reporting checklist (available at http://dx.doi.org/10.21037/ jgo-20-277).

\section{Methods}

We performed 52 cases of total laparoscopic total and proximal gastrectomy consecutively from January
2018 to May 2020, all of which were performed by intraoperative gastroscopy (EVIS-180; Olympus) to locate the proximal resection margin. All patients received TLG reconstruction by Roux-en-Y with linear stapled methods or esophagogastrostomy for gastric cancer and histologically proven adenocarcinoma at the Beijing Cancer Hospital (Beijing, China). In total, 17 patients received preoperative treatment; of them, thirteen received neoadjuvant treatments, such as chemotherapy or combined radiotherapy and chemotherapy, and four underwent endoscopic submucosal dissection that require salvage surgery beyond normal indications. None of the included patients required additional preoperative gastroscopy for tumor localization. Clinicopathological characteristics studied included age, gender, operation time, tumor size, resection type, histology, surgical margin status, depth of tumor, lymph node status, lymphatic vascular infiltration (LVI), Siewert classification, EGJ invasion and neoadjuvant treatment. Tumors were staged in accordance with the American Joint Committee on Cancer (AJCC eighth edition). According to this system, patients receiving neoadjuvant treatment who have a complete pathologic response following surgery are classified as stage 0 . Informed consent was obtained from all patients before surgery, and clinical and pathological data were retrieved from the medical notes. The primary outcome of this study was the percentage of resection positive margins. The study was conducted in accordance with the Declaration of Helsinki (as revised in 2013). The study was approved by the Institutional Review Board of Peking University Cancer Hospital (No. 2020KT02) and informed consent was taken from all the patients.

\section{Intraoperative endoscopy}

Two doctors from the endoscopic center participated in the intraoperative endoscopy work, and they alternated every month. During the surgeries, accurate measurements and positioning were done using a scale placed under the endoscope. One of the endoscopists defined the normal mucosa $1 \mathrm{~cm}$ from the proximal edge of the tumor, and the other defined the normal mucosa $2 \mathrm{~cm}$ from the proximal edge of the tumor according to the different preferences of endoscopists

Operating rooms have access to equipment dedicated for intraoperative gastroscopy use. The procedure differed somewhat from that of preoperative gastroscopy. The patient was placed in the supine position under general anesthesia. Gastroscopic findings were simultaneously 


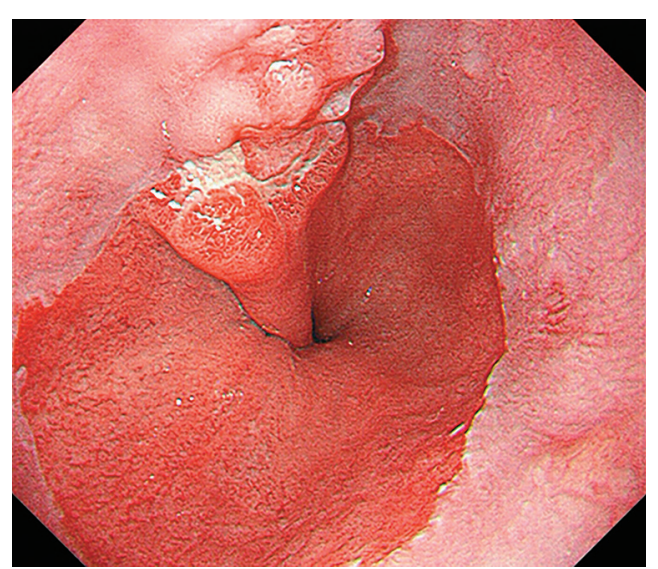

Figure 1 The proximal tumor is easily identified under intraoperative gastroscopy (EGJ invasion).
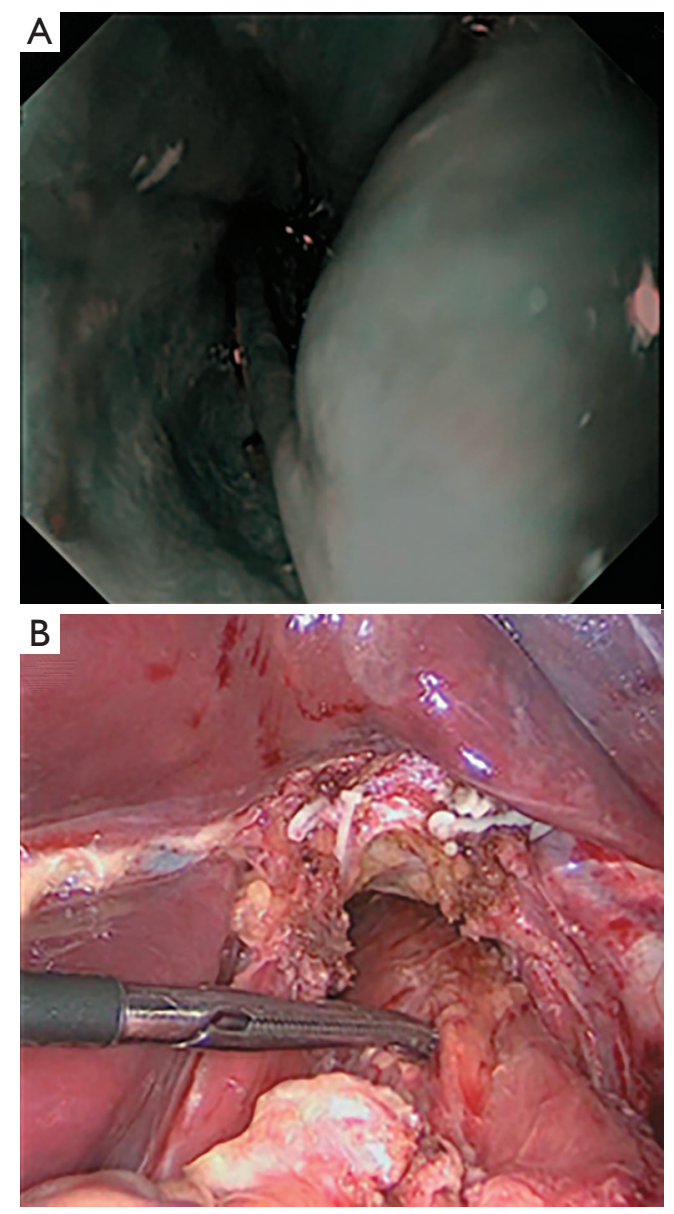

Figure 2 In the endoscopic view, the location of the proximal tumor margin is compared with that of the mucosal protrusion formed by laparoscopic manipulation: (A) endoscopic view and (B) laparoscopic view. available to the endoscopist and surgeon by monitor. Lymph nodes surrounding the stomach were dissected laparoscopically, and the duodenum was transected $1-2 \mathrm{~cm}$ distal to the pylorus using an endoscopic stapling device. After that was completed, one gastrointestinal endoscopist performed the intraoperative endoscopy. When the surgeon prepared to resect the esophagus, endoscopy with carbon dioxide insufflation was then performed by the endoscopist (Figure 1). After confirming the normal mucosa through the gastroscopy, the surgeon detected lesions by pushing down the gastric wall with forceps and identifying the matching mucosal protrusion on the gastroscopy (Figure 2). The endoscopist decided the proximal location of the tumor in the endoscopic view, and the surgeon determined the proximal location of the tumor and marked it with a marker in the laparoscopic view (Figure 3). Then, the esophagus was clamped using a linear stapler. Before the stapler was fired, gastroscopy was performed to confirm that the tumor was not visible (Figure 4). After the stapling, the abdominal esophagus was transected. The surgeon resected the stomach under the guidance of intraoperative gastroscopy in proximal gastrectomy.

\section{Pathological analysis}

As soon as the stomach was resected, specimens were placed in an endoscopic bag and extracted from the abdominal cavity through a $2-3 \mathrm{~cm}$ extension of the infraumbilical trocar site. Adequate tumor margins were macroscopically confirmed, and tissue from the proximal margin was frozen for intraoperative histological evaluation. After confirmation of tumor-free tissue in the proximal portion of the EGJ, an anastomosis between the esophagus and the proximal jejunum was formed with intracorporeal linear staples. Histopathological analysis of formalin-fixed, paraffinembedded tissue sections was performed to examine all resected specimens. Tumor depth, pathological type, and LVI were characterized.

\section{Statistical analysis}

All statistical analyses were conducted using SPSS for Windows version 22.0 (Chicago, IL, USA). Categorical variables were analyzed using the chi-square or Fisher's exact test. Logistic regression analysis was used to determine the independent risk factors for the presence of positive resection margin. All tests were two-sided, and $\mathrm{P}<0.05$ was 


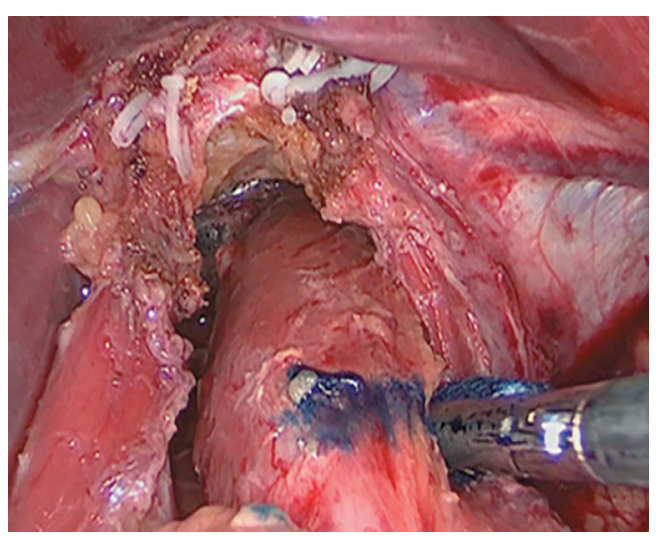

Figure 3 Marking the external surface of the esophagus in the laparoscopic view.

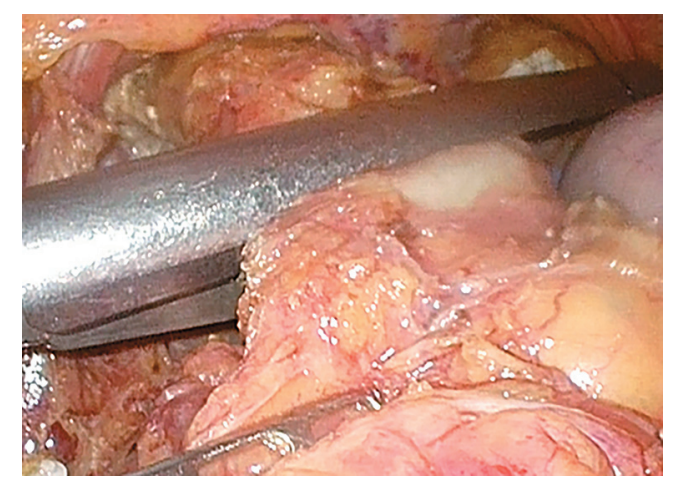

Figure 4 The esophagus is clamped using a linear stapler before firing it, and gastroscopy confirmed that the tumor was not visible.

considered statistically significant difference.

\section{Results}

The clinicopathological characteristics of patients are shown in Table 1. In total, 42 patients had Siewert type II tumors and 10 had Siewert type III. The median age of the patients (41 males and 11 females) was 63.5 years (range, 36-80 years). On the basis of intraoperative gastroscopic findings, total gastrectomy was performed in 35 patients, and 17 patients received proximal gastrectomy. The median tumor size was $3 \mathrm{~cm}$ (range, $1-7 \mathrm{~cm}$ ). The median TLG operative time was $244 \mathrm{~min}$ (range, 143-480 $\mathrm{min}$ ). The median time required for tumor localization with intraoperative gastroscopy was 5.8 min. Lymph node metastasis was observed in 12 cases. Tumors involving the EGJ were seen in 27 patients. There were no complications related to intraoperative gastroscopy.
The proximal portion of tumors was confirmed by intraoperative gastroscopy in all patients. The patients were divided into short $(1 \mathrm{~cm})$ and long $(2 \mathrm{~cm})$ groups according to the distance to the proximal margin of the tumor by the different endoscopists; there were 23 patients in the short group and 29 in the long group. The median of actual gross proximal margin lengths was $2 \mathrm{~cm}$ in the long group and $0.8 \mathrm{~cm}$ in the short group, respectively. As shown in Table 2, there were no significant differences between the short and long groups in terms of sex, age, Lauren pattern, operation time, resection type (total $v s$. proximal), neoadjuvant chemotherapy or combined radiotherapy and chemotherapy, histology, depth of tumor, lymph node status, Siewert classification, or EGJ invasion. The long group had a frequency of positive margin that was statistically significantly lower than that of the short group ( $0 \%$ vs. $17.4 \%, \mathrm{P}=0.033$ ). The calculated OR for positive margin in the case of the short group was 4.7 (95\% CI: 1.9-32.9; $\mathrm{P}=0.022)$. Four patients in the short group had a positive margin by frozen section analysis during surgery. Thus, the surgeon resected the esophagus for examination again; subsequently, the gastric resections were successfully performed with no positive margin as confirmed by frozen section examination during surgery. All tumor resection margins were also tumor-negative after histopathological examination of formalin-fixed, paraffin-embedded tissues. Four patients achieved a complete pathologic response after receiving neoadjuvant treatment, and four patients were scheduled for salvage surgery following noncurative endoscopic submucosal dissection, of which one patient still had residual tumor after surgery.

According to the postoperative pathological staging, the resection margins in patients with pT0-1were negative (0/28). Further subgroup analysis shows the long group had a statistically significant lower frequency of positive margin than the short group ( $0 \%$ vs. $40 \%$, $\mathrm{P}=0.020$ ) in patients with $\mathrm{pT} 2-4$. There were no significant differences between the positive and negative margin groups in terms of sex, age, Lauren pattern, operation time, resection type (total $v$ s. proximal), neoadjuvant chemotherapy or combined radiotherapy and chemotherapy, histology, tumor stage, Siewert classification, or EGJ invasion (Table 3).

\section{Discussion}

The number of reports on laparoscopy-assisted total gastrectomy for gastric cancer has increased with the 
Table 1 The clinicopathological characteristics of 52 patients undergoing totally laparoscopic gastrectomy

\begin{tabular}{|c|c|}
\hline Variables & Values \\
\hline Age, years & 63.5 \\
\hline \multicolumn{2}{|l|}{ Sex, n (\%) } \\
\hline Male & $41(79.2)$ \\
\hline Female & $11(20.8)$ \\
\hline Operation time, $\min$ & 244 \\
\hline \multicolumn{2}{|l|}{ Resection type, n (\%) } \\
\hline Total & $35(67.3)$ \\
\hline Proximal & $17(32.7)$ \\
\hline \multicolumn{2}{|l|}{ Lauren pattern, n (\%) } \\
\hline Intestinal & $30(57.6)$ \\
\hline Diffuse/mixed & $11(21.2)$ \\
\hline Other & $11(21.2)$ \\
\hline \multicolumn{2}{|l|}{ Tumor size, n (\%) } \\
\hline$>2 \mathrm{~cm}$ & $26(50.0)$ \\
\hline$\leq 2 \mathrm{~cm}$ & $26(50.0)$ \\
\hline \multicolumn{2}{|l|}{ Neoadjuvant treatment, n (\%) } \\
\hline Yes & $13(25.0)$ \\
\hline No & $39(75.0)$ \\
\hline \multicolumn{2}{|l|}{ After ESD, n (\%) } \\
\hline Yes & $4(7.7)$ \\
\hline No & $48(92.3)$ \\
\hline \multicolumn{2}{|l|}{ Histology, n (\%) } \\
\hline Differentiated & $35(67.3)$ \\
\hline Undifferentiated & $7(13.5)$ \\
\hline No tumor or degenerative cancer cells & $6(11.5)$ \\
\hline Adenosquamous carcinoma & $3(5.8)$ \\
\hline Hepatoid adenocarcinoma & $1(1.9)$ \\
\hline \multicolumn{2}{|l|}{ Depth of tumor, n (\%) } \\
\hline 0 & $4(7.7)$ \\
\hline pT1a & $12(23.1)$ \\
\hline pT1b & $12(23.1)$ \\
\hline pT2 & $8(15.5)$ \\
\hline pT3 & $14(26.9)$ \\
\hline
\end{tabular}

Table 1 (continued)
Table 1 (continued)

\begin{tabular}{lc}
\hline Variables & Values \\
\hline pT4 & $2(3.8)$ \\
Lymph node status, $\mathrm{n}(\%)$ & \\
0 & $40(76.9)$ \\
$\mathrm{pN} 1$ & $5(9.6)$ \\
$\mathrm{pN} 2$ & $1(1.9)$ \\
$\mathrm{pN} 3 \mathrm{a}$ & $6(11.6)$ \\
LVI, $\mathrm{n}$ (\%) & \\
Yes & $15(28.8)$ \\
No & $37(71.2)$ \\
Siewert classification, $\mathrm{n}(\%)$ & \\
Siewert II & $42(80.8)$ \\
Siewert III & $10(19.2)$ \\
EGJ invasion, $\mathrm{n}(\%)$ & \\
Yes & $27(51.9)$ \\
No & $25(48.1)$ \\
\hline
\end{tabular}

advancement of techniques for lymphadenectomy and reconstructive procedures for the upper stomach. However, esophagojejunostomy via minilaparotomy in laparoscopyassisted total gastrectomy is relatively difficult because of the limited angle of the direct view, depending on the patient's somatotype and obesity index (19-21). With the development of techniques for the construction of an intracorporeal anastomosis during laparoscopic gastrectomy, TLG has become more common worldwide $(4,17,18)$. Unfortunately, the best method for determining proximal tumor resection margins, especially for early cancer located in the upper and EGJ region, remains unclear, and resection with a safe margin in advanced cases is difficult in TLG.

At present, the gold standard for determining the esophageal edge is frozen section diagnosis during surgery. In laparotomy- or laparoscopic-assisted surgery, the EGJ can be palpated to roughly judge the proximal edge of the tumor, while frozen section pathology enables judgment of whether there was tumor invasion. However, the ability to palpate the stomach is lost during TLG; therefore, this technique cannot accurately and reliably determine the range of resection in the upper third of the stomach, especially for EGJ invasion. Under intraoperative 
Table 2 Comparison of demographic, clinical, and histopathological characteristics between short group and long group

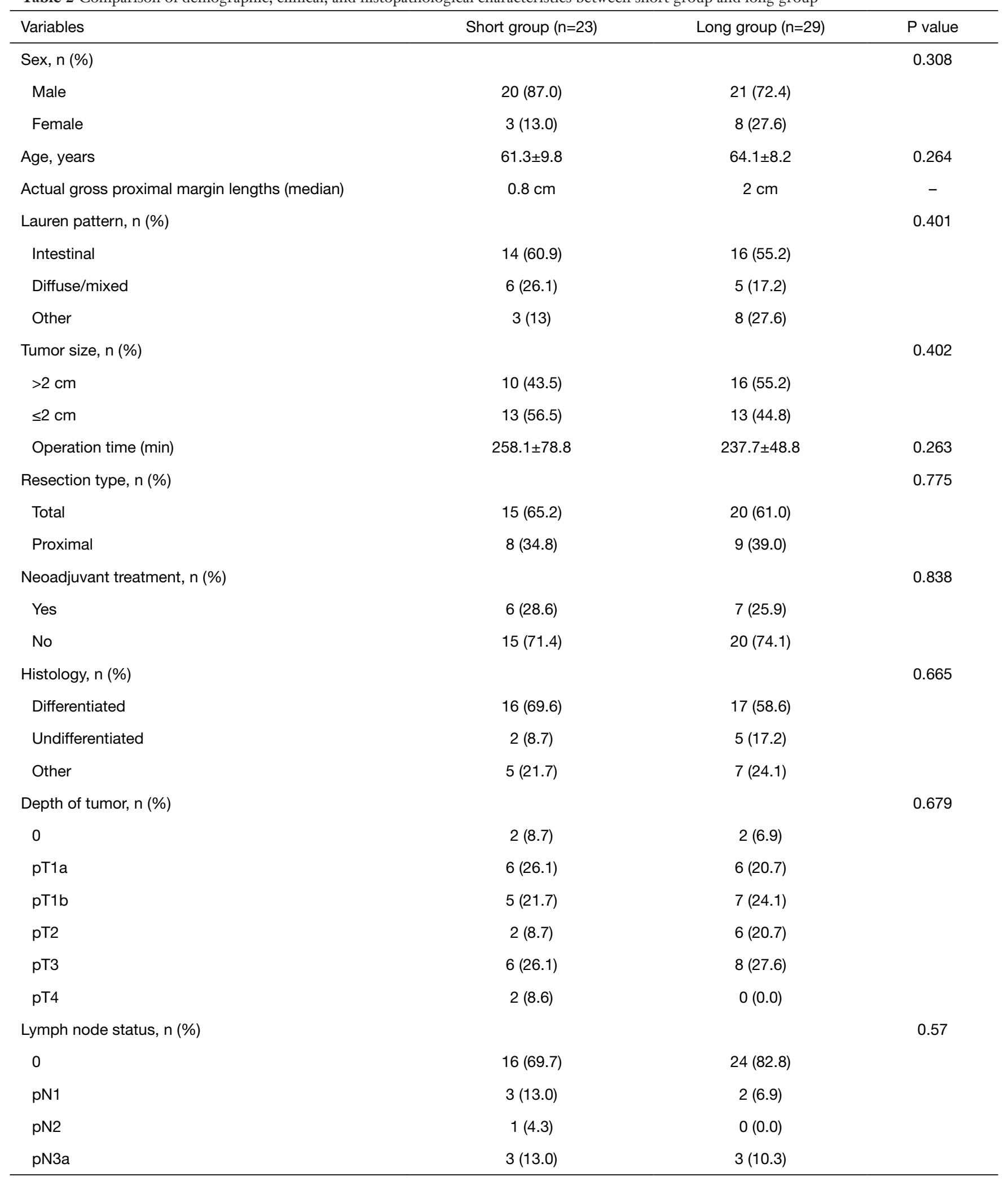

Table 2 (continued) 
Table 2 (continued)

\begin{tabular}{lcc}
\hline Variables & Short group $(\mathrm{n}=23)$ & Long group $(\mathrm{n}=29)$ \\
\hline LVI, $\mathrm{n}(\%)$ & $6(26.1)$ & $9(31.0)$ \\
Yes & $17(73.9)$ & $20(69.0)$ \\
No & & 0.696 \\
Siewert classification, $\mathrm{n}(\%)$ & $18(78.3)$ & $20(69.0)$ \\
Siewert II & $5(21.7)$ & $9(31.0)$ \\
Siewert III & & $12(41.4)$ \\
EGJ invasion, $\mathrm{n}(\%)$ & $15(65.2)$ & $17(58.6)$ \\
Yes & $8(34.8)$ & 0.087 \\
No & & 0.453 \\
Positive margin, $\mathrm{n}(\%)$ & $4(17.4)$ & $29(100.0)$ \\
Yes & $19(82.6)$ & 0.033 \\
No & & 2 \\
\hline
\end{tabular}

endoscopy guidance, more abdominal portions of the esophagus may be preserved, which would be convenient for esophagojejunostomy or esophagogastrostomy because the linear staplers used in TLG require a substantial length of the mobilized esophagus for anastomosis.

To date, several techniques have been applied to identify the location of tumors in the laparoscopic view, such as preoperative or intraoperative tattooing and preoperative metal clipping, wherein the location of clips can be detected by intraoperative ultrasonography (7), radiography $(12,14)$, magnetic marking clip-detecting systems (MMCDS) (16), and intraoperative gastroscopy $(8,22)$. However, all of these methods have limitations, and additional preoperative gastroscopy is required before surgery. In preoperative or intraoperative tattooing, the injected dye (e.g., indocyanine, patent blue, indigo carmine, and blood) can easily diffuse into the serosal surface and obscure the precise tumor location, making it difficult to determine an appropriate resection line (11,13,23-25). Intraperitoneal spillage is also relatively common and could cause difficulties during surgery by staining the operation field and causing subsequent adhesion ileus due to chemical peritonitis. Detection of clips by laparoscopic ultrasonography, which involves subjective interpretation, can require an additional level of skill and experience. Intraoperative portable radiography can only supply two-dimensional views, and it may be difficult to locate a tumor in a stomach that is not distended. Furthermore, patients and physicians may be exposed to radiation. In MMCDS, tumors are localized by an MMCDS probe that responds to magnetic clips attached during preoperative endoscopy (16).

Gastric cancer initially developed from the mucosal and invaded the serosal layers gradually, it was more accurate for the upper edge of the tumor determined by endoscopy. The present study mainly focused on the location of the esophageal margin in TLG. Because an intraoperative gastroscopic view allows exact proximal localization of the tumor, preoperative metal clips or dye injection was not required. After the surgeon separated the esophagus, the esophagus wall can be clearly exposed, under the guidance of the endoscopist, the surgeon can accurately locate the upper resection margin of the tumor (Figure 3). We found that all patients had negative margins as confirmed by frozen sections in the long group, whereas there were four cases with positive margins in the short group, resulting in a significant difference between the groups with respect to positive resection margin incidence $(\mathrm{P}=0.033)$. According to our results, a negative margin can be ensured if the normal tissues were $\geq 2 \mathrm{~cm}$ away from the tumor margin, potentially because the tumor may have submucosal infiltration, which leads to a positive margin, our research also confirmed that the short group was more likely to have positive margins in patients with cT2-4 diseases. After the location of the lesion was confirmed, the esophagus was clamped using an endoscopic linear stapler. The surgeon further ensured the safety of proximal margin resection, as well as 
Table 3 Comparison of demographic, clinical, and histopathological characteristics between positive and negative margin groups

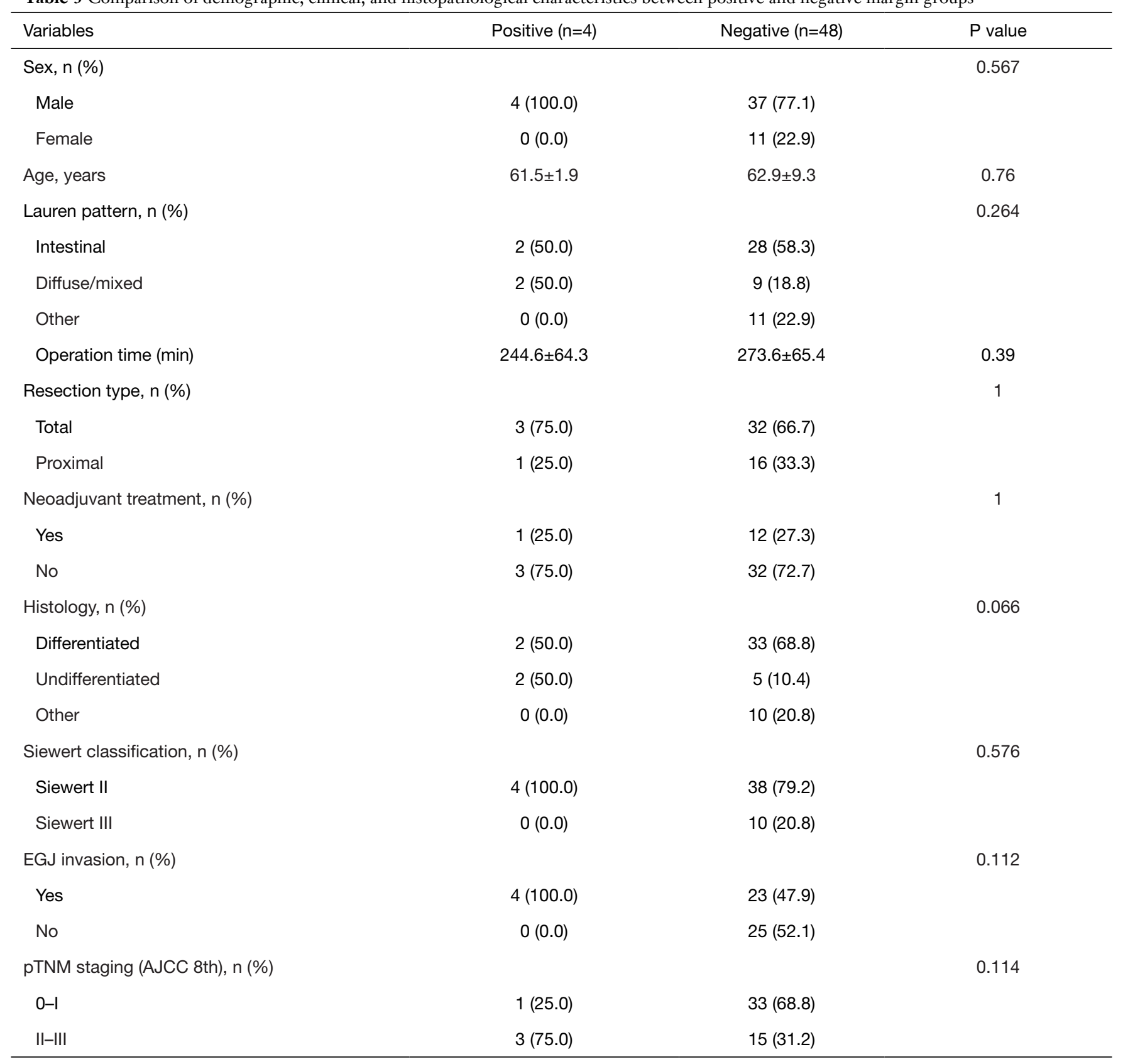

safeguarded against resecting too much esophagus to reduce the difficulty of subsequent esophagojejunostomy. After the tumor specimen was removed, the proximal margin was sent for frozen section pathology examination, and esophagojejunostomy or esophagogastric anastomosis were performed after confirmation of tumor-free tissue in the proximal portion of the EGJ.

Unlike other procedures, gastroscopy was performed under general anesthesia. Therefore, patients enrolled in the present study avoided extra preoperative gastroscopy to locate tumor margin by tattooing or metal clipping. Intraoperative gastroscopy increases the overall operation time because it takes a while for the gastroenterologist and gastroscopic system to arrive. These disadvantages were avoided in the present study as two gastroscopic systems were placed in the operating room to perform intraoperative 
endoscopy, and it was not necessary to ensure the proximal location of the tumor at the start of surgery. After lymph nodes surrounding the stomach and duodenum were laparoscopically dissected, the endoscopist was invited into the operating room. Therefore, the surgical process was not interrupted until the endoscopist arrived. Thus, the median time for tumor localization with intraoperative gastroscopy only was $5.8 \mathrm{~min}$. The endoscopists involved in the present study had sufficient experience $(>5,000$ cases each) with gastroscope manipulation and had worked in the Department of Gastrointestinal Surgery previously during resident training, qualifying them to accurately judge the tumor boundary in close cooperation with experienced surgeons.

Of the 13 patients who received neoadjuvant treatment in the current study, one who received combined preoperative radiotherapy and chemotherapy acquired pathologic complete response. Radiotherapy results in fibrosis of the distal esophageal mucosal layer. Under endoscopic guidance, the esophagus can be transected at the normal esophageal mucosa, reducing the risk of postoperative complications, such as anastomotic leakage or stenosis. Frozen section pathological examination must be completed during surgery in order to ensure the negative margin. However, in future, this may not be necessary unless the proximal margin is suspected of containing some tumor residue during surgery, thereby further shortening the operation time.

There was no positive margin in all patients who did not invade the EGJ whether the long group or the short group. Further subgroup analysis shows that although the long group had a lower frequency of positive margins in the patients involving the EGJ, but the difference was not statistically significant $(0 \%$ vs. $26.7 \%, \mathrm{P}=0.106)$, perhaps due to the limited number of patients included in the study. But it also indicates that the distance of the proximal resection margin maybe beyond $2 \mathrm{~cm}$ from the edge of the tumor in patients with EGJ invasion. Although positive resection margins occurred all in the Siewert type II cancer, but $28.6 \%$ (4/14) of Siewert type III cancer tumors also have EGJ invasion, so it was still needs $\geq 2 \mathrm{~cm}$ proximal margin to obtain pathologically negative margin in patients with Siewert type III cancer.

Several limitations to the present research should be considered. First, this was a single-center, retrospective study with a small number of patients. Second, the cooperating endoscopists who participated had at least 5 years of professional surgical training from the Department of Endoscopy, and the working relationship between the Department of Gastrointestinal Surgery and the Department of Endoscopy at Beijing Cancer Hospital is very close. However, in other hospitals, such relationships may not exist or be strong enough, and it may be difficult to perform intraoperative endoscopy whenever possible. Third, the tumor may have esophageal submucosal infiltration, making the margin difficult to detect by endoscopy. In this study, we found that the margin maybe safe if the normal esophagus mucosa was dissected $\geq 2 \mathrm{~cm}$ away from the proximal of the tumor margin, but confirmation by frozen section examination still be required, further research is needed to confirm whether intraoperative endoscopic findings can replace frozen section diagnosis.

\section{Conclusions}

In conclusion, coordination of laparoscopic and intraoperative endoscopic views for tumor proximal margin localization was an accurate and useful method for patients with upper third gastric cancer undergoing TLG and avoided additional time-consuming procedures. Our results indicated that the distance from the proximal resection margin to the tumor should be $\geq 2 \mathrm{~cm}$ to achieve a negative resection margin, especially for patients with cT2-4 diseases. However, a comparative trial should be undertaken to confirm the effectiveness of this technique. Nonetheless, it is thought that the clinical value of intraoperative endoscopy in laparoscopic gastrointestinal surgery will increase; it may be prepared as an alternative to frozen section diagnosis in future.

\section{Acknowledgments}

Thank you for Enago editing service.

Funding: This work was supported by Beijing Municipal Administration of Hospitals Incubating Program (PX 2020047), National natural science foundation of China (31870805) and Beijing Municipal Science \& Technology Commission (Z171100001017135).

\section{Footnote}

Reporting Checklist: The authors have completed the STROBE reporting checklist. Available at http://dx.doi. org/10.21037/jgo-20-277

Data Sharing Statement: Available at http://dx.doi. 
org/10.21037/jgo-20-277

Peer Review File: Available at http://dx.doi.org/10.21037/ jgo-20-277

Conflicts of Interest: All authors have completed the ICMJE uniform disclosure form (available at http://dx.doi. org/10.21037/jgo-20-277). The authors have no conflicts of interest to declare.

Ethical Statement: The authors are accountable for all aspects of the work in ensuring that questions related to the accuracy or integrity of any part of the work are appropriately investigated and resolved. The study was conducted in accordance with the Declaration of Helsinki (as revised in 2013). The study was approved by the Institutional Review Board of Peking University Cancer Hospital (No. 2020KT02) and informed consent was taken from all the patients.

Open Access Statement: This is an Open Access article distributed in accordance with the Creative Commons Attribution-NonCommercial-NoDerivs 4.0 International License (CC BY-NC-ND 4.0), which permits the noncommercial replication and distribution of the article with the strict proviso that no changes or edits are made and the original work is properly cited (including links to both the formal publication through the relevant DOI and the license). See: https://creativecommons.org/licenses/by-nc-nd/4.0/.

\section{References}

1. McAuliffe JC, Tang LH, Kamrani K, et al. Prevalence of False-Negative Results of Intraoperative Consultation on Surgical Margins During Resection of Gastric and Gastroesophageal Adenocarcinoma. JAMA Surg 2019;154:126-32.

2. Bissolati M, Desio M, Rosa F, et al. Risk factor analysis for involvement of resection margins in gastric and esophagogastric junction cancer: an Italian multicenter study. Gastric Cancer 2017;20:70-82.

3. Liang Y, Ding X, Wang X, et al. Prognostic value of surgical margin status in gastric cancer patients. ANZ J Surg 2015;85:678-84.

4. Zhao S, Zheng K, Zheng JC, et al. Comparison of totally laparoscopic total gastrectomy and laparoscopic-assisted total gastrectomy: A systematic review and meta-analysis. Int J Surg 2019;68:1-10.
5. Han $\mathrm{WH}$, Yehuda AB, Kim DH, et al. A comparative study of totally laparoscopic distal gastrectomy versus laparoscopic-assisted distal gastrectomy in gastric cancer patients: Short-term operative outcomes at a high-volume center. Chin J Cancer Res 2018;30:537-45.

6. Wang H, Mou T, Chen H, et al. Long-term outcomes of laparoscopy-assisted distal gastrectomy versus open distal gastrectomy for gastric cancer: a 10-year single-institution experience. Surg Endosc 2019;33:135-44.

7. Matsuda T, Iwasaki T, Hirata K, et al. Simple and reliable method for tumor localization during totally laparoscopic gastrectomy: intraoperative laparoscopic ultrasonography combined with tattooing. Gastric Cancer 2017;20:548-52.

8. Kawakatsu S, Ohashi M, Hiki N, et al. Use of endoscopy to determine the resection margin during laparoscopic gastrectomy for cancer. Br J Surg 2017;104:1829-36.

9. Lee CM, Park S, Park SH, et al. A comparison between two methods for tumor localization during totally laparoscopic distal gastrectomy in patients with gastric cancer. Ann Surg Treat Res 2016;91:112-7.

10. Kim SH, Keum B, Jeen YT, et al. Efficacy of the magnetic marking clip method for preoperative localization of GI tumor. Gastrointest Endosc 2016;83:468-9.

11. Nakagawa M, Ehara K, Ueno M, et al. Accurate, safe, and rapid method of intraoperative tumor identification for totally laparoscopic distal gastrectomy: injection of mixed fluid of sodium hyaluronate and patent blue. Surg Endosc 2014;28:1371-5.

12. Kim BS, Yook JH, Kim BS, et al. A simplified technique for tumor localization using preoperative endoscopic clipping and radio-opaque markers during totally laparoscopic gastrectomy. Am Surg 2014;80:1266-70.

13. Jeong $\mathrm{O}$, Cho SB, Joo YE, et al. Novel technique for intraoperative tumor localization during totally laparoscopic distal gastrectomy: endoscopic autologous blood tattooing. Surg Endosc 2012;26:1778-83.

14. Kim HI, Hyung WJ, Lee CR, et al. Intraoperative portable abdominal radiograph for tumor localization: a simple and accurate method for laparoscopic gastrectomy. Surg Endosc 2011;25:958-63.

15. Matsui H, Okamoto Y, Nabeshima K, et al. Endoscopyassisted gastric resection: a safe and reliable procedure for tumor clearance during laparoscopic high distal or proximal gastrectomy. Surg Endosc 2009;23:1146-9.

16. Ohdaira T, Nagai H. Intraoperative localization of earlystage upper gastrointestinal tumors using a magnetic marking clip-detecting system. Surg Endosc 2007;21:810-5.

17. Murakami K, Obama K, Tsunoda S, et al. Linear or 
circular stapler? A propensity score-matched, multicenter analysis of intracorporeal esophagojejunostomy following totally laparoscopic total gastrectomy. Surg Endosc 2020;34:5265-73.

18. Lee SW, Kawai M, Tashiro K, et al. The crossover technique for intracorporeal esophagojejunostomy following laparoscopic total gastrectomy: a simple and safe technique using a linear stapler and two barbed sutures. Surg Endosc 2019;33:1386-93.

19. Liu D, Liang L, Liu L, et al. Short-term outcomes and prognosis of laparoscopy-assisted total gastrectomy in elderly patients with stomach cancer. Surg Endosc 2020;34:5428-38.

20. Katai H, Mizusawa J, Katayama H, et al. Single-arm confirmatory trial of laparoscopy-assisted total or proximal gastrectomy with nodal dissection for clinical stage I gastric cancer: Japan Clinical Oncology Group study JCOG1401. Gastric Cancer 2019;22:999-1008.

21. Lee JH, Nam BH, Ryu KW, et al. Comparison

Cite this article as: Yuan P, Yan Y, Jia Y, Wang J, Li Z, Wu Q. Intraoperative gastroscopy to determine proximal resection margin during totally laparoscopic gastrectomy for patients with upper third gastric cancer. J Gastrointest Oncol 2021;12(1):142-152. doi: 10.21037/jgo-20-277 of outcomes after laparoscopy-assisted and open total gastrectomy for early gastric cancer. Br J Surg 2015;102:1500-5.

22. Gunji H, Horibe D, Uesato M, et al. Gastric Resection Under Retroflexed Endoscopic Guidance: A Reliable Procedure for Totally Laparoscopic Subtotal Gastrectomy. Dig Surg 2017;34:12-7.

23. Ushimaru Y, Omori T, Fujiwara Y, et al. The Feasibility and Safety of Preoperative Fluorescence Marking with Indocyanine Green (ICG) in Laparoscopic Gastrectomy for Gastric Cancer. J Gastrointest Surg 2019;23:468-76.

24. Hur H, Son SY, Cho YK, et al. Intraoperative Gastroscopy for Tumor Localization in Laparoscopic Surgery for Gastric Adenocarcinoma. J Vis Exp 2016;(114):53170.

25. Xuan Y, Hur H, Byun CS, et al. Efficacy of intraoperative gastroscopy for tumor localization in totally laparoscopic distal gastrectomy for cancer in the middle third of the stomach. Surg Endosc 2013;27:4364-70. 